\title{
Clinical characteristics and prognosis of basaloid squamous cell carcinoma of the lung: a population-based analysis
}

\author{
Guangda Yuan ${ }^{1}$, Cheng Zhan ${ }^{2}$, Yiwei Huang ${ }^{2}$, Donglin Zhu ${ }^{1}$, Hongya Xie ${ }^{1}$, Tengteng Wei ${ }^{1}$, Tao Lu ${ }^{2}$, \\ Qun Wang ${ }^{2}$, Yong Yang ${ }^{\text {Corresp., }}{ }^{1}$, Yimeng Zhu ${ }^{\text {Corresp. } 1}$ \\ 1 Department of Thoracic Surgery, The Affiliated Suzhou Hospital of Nanjing Medical University, Suzhou, Jiangsu, China \\ 2 Department of Thoracic Surgery, Zhongshan Hospital, Fudan University, Shanghai, China \\ Corresponding Authors: Yong Yang, Yimeng Zhu \\ Email address: yangy_suzhou@126.com, zhuym_suzhou@126.com
}

Background: This study analyzed the clinical features and prognosis of basaloid squamous cell carcinoma of the lung (BSC), and constructed a nomogram to predict the prognoses of patients. Methods: The information of pure BSC patients was obtained in the Surveillance, Epidemiology, and End Results database between 2004 and 2015. Then, it was evaluated, and compared with the data of lung squamous cell carcinoma (SCC), lung large cell carcinoma (LCC) and lung adenocarcinoma (LAC) patients. Subsequently, we used univariate and multivariate analyses to investigate the independent factors related to the prognoses of patients with BSC and constructed a nomogram to verify the prognoses.

Results: A total of 425 patients diagnosed with BSC were enrolled. Compared with patients with SCC, LCC and LAC, the mean survival time of BSC patients was better than all of them. Compared with SCC, there were significant difference between the characteristics of grade $(P<0.001)$, total stage $(P<0.001)$, T stage $(P<0.001)$, $N$ stage $(P<0.001)$, M stage $(P<0.001)$, surgery $(P<0.001)$, radiotherapy $(P<0.001)$, and chemotherapy $(P<0.001)$, while BSC also had significantly different clinical characteristics from LCC and LAC. Univariate and multivariate survival analyses showed that age $(P<0.001)$, T stage $(P<0.001), N$ stage $(P=0.009)$, $M$ stage $(P<0.001)$, and surgery $(P<0.001)$ were independent prognostic factors of BSC. The survival of patients undergoing lobectomy was significantly better than sublobar resection, with an OR of 0.389 (0.263-0.578). We constructed a nomogram with a C-index of 0.750 (95\% confidence interval) based on the results of multivariate analysis. The calibration curves based on nomogram scores indicated that the nomogram could accurately predict the prognosis of patients. Conclusions: BSC had unique clinical and prognostic features. T stage, $\mathrm{N}$ stage, $\mathrm{M}$ stage, age, and surgery were independently associated with overall survival (OS). Lobectomy was a relative ideal choice for patients with BSC. The nomogram effectively predicted the OS at 1-, 3-, and 5-years. 


\section{Clinical characteristics and prognosis of basaloid squamous cell carcinoma of}

\section{2 the lung: a population-based analysis}

3 Guangda Yuan ${ }^{1 *}$, Cheng Zhan ${ }^{2 *}$, Yiwei Huang ${ }^{2 *}$, Donglin Zhu ${ }^{1}$, Hongya Xie ${ }^{1}$, Tengteng Wei ${ }^{1}$,

4 Tao Lu², Qun Wang ${ }^{2}$, Yong Yang ${ }^{1} \&$ Yimeng $\mathrm{Zhu}^{1}$

51 Department of Thoracic Surgery, the Affiliated Suzhou Hospital of Nanjing Medical University, Suzhou,

6 Jiangsu, China

72 Department of Thoracic Surgery, Zhongshan Hospital, Fudan University, Shanghai, China

$8 \quad$ * Guangda Yuan, Cheng Zhan and Yiwei Huang contributed equally to this work.

\section{Correspondence}

10 Yong Yang \& Yimeng Zhu, Department of Thoracic Surgery, The Affiliated Suzhou Hospital of Nanjing

11 Medical University, Suzhou, Jiangsu, China.

12 Tel: 8651262364519

13 Fax: 8651262364519

14 Email: zhuym suzhou@126.com

15 yangy_suzhou@126.com

\section{Abstract}

17 Background: This study analyzed the clinical features and prognosis of basaloid squamous cell carcinoma of the lung (BSC), and constructed a nomogram to predict the prognoses of patients.

Methods: The information of pure BSC patients was obtained in the Surveillance, Epidemiology, and End 
22 Subsequently, we used univariate and multivariate analyses to investigate the independent factors related to the prognoses of patients with BSC and constructed a nomogram to verify the prognoses.

Results: A total of 425 patients diagnosed with BSC were enrolled. Compared with patients with SCC, LCC and LAC, the mean survival time of BSC patients was better than all of them. Compared with SCC, there were significant difference between the characteristics of grade $(\mathrm{P}<0.001)$, total stage $(\mathrm{P}<0.001)$, $\mathrm{T}$ stage $(\mathrm{P}<0.001)$, $\mathrm{N}$ stage ( $\mathrm{P}<0.001)$, M stage $(\mathrm{P}<0.001)$, surgery $(\mathrm{P}<0.001)$, radiotherapy $(\mathrm{P}<0.001)$, and chemotherapy $(\mathrm{P}<0.001)$, while BSC also had significantly different clinical characteristics from LCC and LAC. Univariate and multivariate survival analyses showed that age $(\mathrm{P}<0.001), \mathrm{T}$ stage $(\mathrm{P}<0.001), \mathrm{N}$ stage $(\mathrm{P}=0.009), \mathrm{M}$ stage $(\mathrm{P}<0.001)$, and surgery $(\mathrm{P}<0.001)$ were independent prognostic factors of $\mathrm{BSC}$. The survival of patients undergoing lobectomy was significantly better than sublobar resection, with an OR of $0.389(0.263-0.578)$. We constructed a nomogram with a C-index of 0.750 (95\% confidence interval) based on the results of multivariate analysis. The calibration curves based on nomogram scores indicated that the nomogram could accurately predict the prognosis of patients.

Conclusions: BSC had unique clinical and prognostic features. $\mathrm{T}$ stage, $\mathrm{N}$ stage, $\mathrm{M}$ stage, age, and surgery were independently associated with overall survival (OS). Lobectomy was a relative ideal choice for patients with BSC. The nomogram effectively predicted the OS at 1-, 3-, and 5-years.

Introduction

Lung cancer is the most commonly diagnosed cancer worldwide, as well as the leading cause of cancer deaths. 
43 Worldwide in 2018, it accounted for 2.1 million new cases and 1.8 million deaths. Squamous cell carcinoma is

44 one of the major well-studied histological subtypes of lung cancer [1, 2]. However, basaloid squamous cell

45 carcinoma of the lung (BSC), as a rare subtype of lung squamous cell carcinoma, is less studied, and the

46 clinical features and prognostic factors remain unclear.

47 BSC is an uncommon histological variant of lung cancer composed of cells exhibiting cytological and tissue architectural features of both squamous cell lung carcinoma and basal cell carcinoma, while the proportion of squamous cell components is less than $50 \%{ }^{[3-5]}$. It is reported that BSC accounts for $3.9 \%-5.2 \%$ of all lung squamous carcinomas and has unique clinical characteristics such as a high rate of metastasis and death, according to previous research. Apart from this, BSC was once described to with overlap features of large cell carcinoma (LCC) ${ }^{[6,7]}$. Up to date, no relevant literatures have reported the difference of clinical features with LCC and other non-small cell lung cancer (NSCLC). In this study, we compared clinicopathological characteristics of related lung cancer subtypes in detail, then we used univariate and Cox hazards regression analyses to identify risk factors affecting overall survival (OS) of BSC. We further developed a nomogram of patients with BSC based on the results of survival analysis to better predict the prognoses of patients.

\section{Materials and Methods}

\section{Ethic statement}

We obtained permission to use data files from the public database of the Surveillance, Epidemiology, and End Results (SEER) database. Thus, our research was exempted by the Ethics Committee of Suzhou Municipal Hospital.

\section{Data extraction}


were identified by the SEER*Stat software (v8.3.5, https://seer.cancer.gov/seerstat/) from the SEER database (http://seer.cancer.gov/). Exclusion criteria were: 1) pathological types of non-pure-type basaloid squamous cell carcinoma; 2) unknown aspects regarding differentiation, stage, and treatment methods; and 3) a history of tumors in other sites (Figure 1).

We extracted and analyzed the data regarding patients' race, sex, age, grade, TMN stage, surgery type, radiotherapy, and chemotherapy. The total stage, $\mathrm{T}$ stage, $\mathrm{N}$ stage, and $\mathrm{M}$ stage of all patients were manually restaged according to the 8th edition of the American Joint Committee on Cancer (AJCC) lung cancer staging project.

Chi-square tests were used for comparison of multi-class variables like race between basaloid squamous cell carcinoma and other types of lung carcinomas. Rank sum tests were used for comparing two categorical variables or ordered variables. The quantitative variables of age were compared by the variance analysis method. In the analysis of prognostic factors for BSC, we used Kaplan-Meier analyses and log-rank tests for univariate analyses, and Cox model tests for multivariate analyses. The above analyses were performed using SPSS (version 25) software (SPSS, Chicago, IL, USA), all of which were two-sided tests, while $\mathrm{P}<0.05$ was considered to be statistically significant. R language (v4.3.4) was used to generate and validate the nomogram, while the main packages used were rms and Hmisc ${ }^{[8,9]}$.

\section{Results}

\section{Comparison of clinical features between BSC and squamous cell carcinoma (SCC)}

After screening, we enrolled 425 patients with BSC of pure type and 90006, 6997 and 160638 patients with SCC, LCC and LAC, respectively. Survival curves indicated that the survival of BSC patients was significantly better than those of SCC, LCC and LACpatients (Figure 2 and Figure 5 of supplemental 
materials). As shown in Table 1, 257 males and 168 females were included in the BSC group, with a median age of 70.15 years and interquartile range (IQR) of $59.87-80.43$ years. In total, $358(84.2 \%)$ were white. The grades included $0.5 \%$ grade I (well differentiated), 10.8\% grade II (moderately differentiated), $64.5 \%$ grade III (poorly differentiated), and 3.0\% grade IV (undifferentiated). Most BSC patients (42.1\%) were AJCC stage I, $14.8 \%$ were stage II, $23.8 \%$ were stage III, and 19.3\% were stage IV. BSC patients had significantly less well differentiated tumors $(\mathrm{P}<0.001)$, less $\mathrm{N}+$ disease $(\mathrm{P}<0.001)$, fewer distant metastases $(\mathrm{P}<0.001)$, lower proportion of radiotherapy $(\mathrm{P}<0.001)$ and chemotherapy $(\mathrm{P}<0.001)$, but a higher percentage of radical surgical resection $(\mathrm{P}<0.001)$ than those of $\mathrm{SCC}$ and LAC patients. Conversely, only LCC patients had more undifferentiated tumors $(\mathrm{P}<0.001)$, while much lower proportion of surgery $(\mathrm{P}<0.001)$, radiotherapy $(\mathrm{P}<0.001)$ and chemotherapy $(\mathrm{P}<0.001)$ (Table 1$)$.

\section{Analyses of BSC prognostic factors}

We used univariate analyses to investigate possible prognostic factors in patients with BSC. As shown in Table 1, there was a statistically significant correlation between age $(\mathrm{P}<0.001)$, grade $(\mathrm{P}<0.001)$, total stage $(\mathrm{P}<0.001), \mathrm{T}$ stage $(\mathrm{P}<0.001), \mathrm{N}$ stage $(\mathrm{P}<0.001)$, $\mathrm{M}$ stage $(\mathrm{P}<0.001)$, surgery $(\mathrm{P}<0.001)$, radiotherapy $(\mathrm{P}<0.001)$ and chemotherapy $(\mathrm{P}=0.013)$ with prognoses of $\mathrm{BSC}$. In other words, elder age, lower differentiation, and a higher total stage meant the worse prognosis. In addition, as shown in Figure 3A, univariate analyses showed that patients who underwent surgery had better prognoses than patients who did not, and were similar to patients receiving other surgical treatments $(\mathrm{P}<0.001)$. Particularly, the patients with Stage I to Stage IV who underwent lobectomy had better benefits than those undergoing sublobar resection (Figure 3B), including for total stage $(\mathrm{P}<0.0001)$, Stage I $(\mathrm{P}=0.00016)$, Stage II $(\mathrm{P}=0.0012)$, Stage III, and IV (both $\mathrm{P}=0.03$ ) (Figure $3 \mathrm{C}, 3 \mathrm{D}, 3 \mathrm{E})$. Sex $(\mathrm{P}=0.257)$ and race $(\mathrm{P}=0.077)$ for the prognosis of $\mathrm{BSC}$ were not 
106

107

108

109

110

111

112

113

114

115

116

117

118

119

120

121

122

123

124

125

126

statistically significant (Table 2).

The data revealed that the factors of age $(\mathrm{P}<0.001)$, $\mathrm{T}$ stage $(\mathrm{P}<0.001)$, $\mathrm{N}$ stage $(\mathrm{P}=0.009)$, M stage $(\mathrm{P}<0.001)$, and surgery $(\mathrm{P}<0.001)$ with statistical significance using univariate analysis were found to be independent factors according to multivariate analyses (Table 2). Multivariate analyses revealed that the older patient and the higher the TMN stage, the worse the prognosis. Excluding the significant effects of $\mathrm{T} 3(\mathrm{P}=0.003), \mathrm{T} 4$ $(\mathrm{P}<0.001)$ and $\mathrm{N} 2(\mathrm{P}=0.001)$, the remaining $\mathrm{T}$ stage and $\mathrm{N}$ stage of the prognoses of patients were similar. Compared with T1 and N0, the odds ratios (ORs) were T2: 1.240 (0.856-1.797), T3: 1.935 (1.257-2.980), T4: $2.364(1.559-3.585)$, and N1: 1.623(1.018-2.586), N2: 1.905(1.300-2.792), and N3: 1.826(0.951-3.505), respectively. The prognoses of patients undergoing lobectomy were significantly better than other surgical styles, with an OR of $0.389(0.263-0.578)$ compared with nonsurgical or other surgical patients. Unlike the results of univariate analysis, multivariate analysis showed that radiotherapy and chemotherapy were not independent prognostic factors.

\section{Production and inspection of the nomogram}

We successfully constructed a nomogram based on the above independent predictors of patient outcomes (Figure 4A). According to the patients' age, $\mathrm{T}$ stage, $\mathrm{N}$ stage, $\mathrm{M}$ stage, and surgery, we visually calculated the patient's 1- (Figure 4B), 3- (Figure 4C) and 5-year (Figure 4D) survival probabilities. The C-index of this nomogram was 0.750 as determined by the discriminant test. The consistency test showed that the 3-year and

5-year survival rates predicted by the nomogram were in good agreement with the actual 3-year and 5-year survival rates, and the slope of the consistency curve was close to 1 .

\section{Discussion}

We conducted an in-depth analysis of BSC using the patients' data from the SEER database and we found that 
127

128

there were significant statistical differences with SCC, LCC and LAC in terms of race, grade, total stage, T stage, $\mathrm{N}$ stage, $\mathrm{M}$ stage, surgery, radiotherapy and chemotherapy. We also found that age, $\mathrm{T}$ stage, $\mathrm{N}$ stage, $\mathrm{M}$ stage and surgery were independent influencing factors for the prognosis of patients with BSC. We then plotted a nomogram. Consistency detection proved that the nomogram effectively predicted the 1-, 3- and 5year survival probabilities of patients, while the nomogram scores effectively discriminated the patients' survivals.

BSC is an invasive subtype of squamous cell carcinoma that can be detected in the proximal bronchi $[4,10]$. Unlike other previous studies [11], we verified that the prognosis of BSC was better than SCC, LCC and LAC. In this study, we found that the prognosis of BSC in a population-based cohort was better than SCC, LCC, and LAC. However, there are some previous studies reported opposite results to ours ${ }^{[12,13]}$, perhaps due to that the number of cases varied, and most of other studies focused on the patients with surgery. Meanwhile, BSC has a significant lower TNM stage than other lung cancer according to our results.

In this population-based study, BSC and other types of lung cancers had similarities in terms of age. But MoroSibilot D et al reported that BSC patients are older than non-BSC patients ${ }^{[13]}$. Thus, Wang LC et al demonstrated that there was no significant statistical difference of mean age between BSC (58.6 years) and poorly differentiated squamous cell carcinoma $(60.5$ years $)(\mathrm{P}=0.363)^{[4]}$. There were more patients with poorly differentiated BSCs, while the numbers of patients of $\mathrm{N}^{+}$and $\mathrm{M}^{+}$were less than those with $\mathrm{SCC}$, LCC and LAC. In our study, the 5-year survival rate of the BSC patients was close to $17.6 \%$. In other reports, the 5-year survival rate for BSC of stage I and stage II was less than 15\%, much lower than the 5-year survival rate of $47 \%$ for resectable poorly differentiated SCC ${ }^{[14]}$. However, Kim et al. reported that there was no significant difference in the median survival rate between BSC and SCC in patients with stage I, without lymph node 
148

metastasis ${ }^{[10]}$. Moreover, Moro et al. reported that operative modes had no difference between the prognosis of BSC and poorly-differentiated SCC ${ }^{[13]}$. As shown in the Figure 6 of supplemental materials, we compared the differences between the two groups by utilizing the survival curve. It clearly indicated that poorlydifferentiated BSCs had better 5-year prognosis than poorly-differentiated SCCs, which were similar to the overall comparison results of this research. Wang LC et al also revealed that BSC and poorly differentiated squamous cell carcinomas had very similar clinical features, and there were no significant differences in survival rates ${ }^{[4]}$, while in our results the survival of poorly differentiated BSC was superior to that of SCC with the same differentiation. More research should be carried out to validate the results.

Currently, surgery is the best curative treatment in stage I, stage II, and some stage III non-small lung cancers [15]. Thus, lobectomy is still recommended as a preferred treatment for BSC, while more patients with peripheral tumors have undergone sublobar section ${ }^{[16]}$. However, both in our univariate analyses and multivariate analyses, patients with lobectomy had a better prognosis than patients undergoing other therapies. Our results also suggested that at any stage, even stage III and IV, the prognosis of patients with lobectomy was significantly better than those with sublobar section. This may due to the radical lobectomy that reduces the potential risks for relapse and distant metastases of solid tumors [17]. In addition, survival following sublobar section was inferior to lobectomy for stage I non-small cell lung cancer ${ }^{[18]}$. Therefore, further studies with larger cohorts, between lobectomy and sublobar section, especially when classified by histology, should be performed.

Nomogram, as an easily available and measurable tool of statistical prediction, which provides prognostic probability of specific outcomes ${ }^{[19,20]}$. So far, multiple nomograms have been constructed for predicting 
168

169

170

171

172

173

174

175

176

177

178

179

180

181

182

183

184

185

186

187

prognosis of different types of lung cancers ${ }^{[21-23]}$. Thus, it has even been considered more applied than the traditional AJCC TNM staging system in diverse malignancies according to great quantity of previous evidence $^{[24,25]}$. Furthermore, nomograms are especially advisable to deal with individual patients without existing definite clinical guidelines. In general, it seems simple and convenient via utilizing nomograms to predict patients' long-time survival according to their own characteristic.

The latest National Comprehensive Cancer Network recommends that EGFR mutations and other gene mutations should be considered as markers for lung squamous cell carcinoma, especially for non-smokers, small biopsy, or mixed squamous cell carcinoma ${ }^{[26,27]}$. Although the gene mutation status has not been well investigated in BSC, molecularly targeted treatment may still have great potential to be used in the treatment for BSC.

The SEER database is a population-based tumor epidemiology database in the United States, covering about $28 \%$ of the population, including thousands of cases of lung cancers since 1973, therefore the SEER database is of great help in the study of lung cancer and other tumors [28, 29]. By analyzing the cases in the entire population of the SEER database, it is possible to effectively avoid the bias of the patients from the research given by a single institution. Nevertheless, there is often a lack of imaging data, smoking history, gene mutations, tumor markers, and data regarding other detailed treatments, especially chemotherapy regimens in the SEER database. Therefore, the impact of these factors on the prognoses of patients with BSC was not included in our study. These factors may significantly affect the prognoses of the patients.

In our study, we have selected BSC cases that met the requirements as much as possible. But there was still a significant gap with the number of SCC. Though there seemed to be some controversy, it was still determined 
by its specific characteristics. We should further pay close attention to the future prognosis of BSCs. We acknowledge that the article limited the findings to epidemiological analysis and did not set more emphasis on exploring the biology of rare tumors such as molecular mechanism for gene therapy strategy.

\section{Conclusion}

BSC has unique clinical and prognostic features that differ from SCC, LCC and LAC. Age, T stage, N stage,

M stage and surgery were found to be independent predictors of prognoses in patients with BSC. The nomogram we constructed better predicted the patients' 1-, 3-, and 5-year survival probabilities.

\section{References:}

[1]. Allemani, C., Matsuda, T., Di Carlo, V., Harewood, R., Matz, M., Niksic, M., Bonaventure, A., Valkov, M., Johnson, C.J., Esteve, J., Ogunbiyi, O.J., Azevedo, E.S.G., Chen, W.Q., Eser, S., Engholm, G., Stiller, C.A., Monnereau, A., Woods, R.R., Visser, O., Lim, G.H., Aitken, J., Weir, H.K., and Coleman, M.P. 2018. Global surveillance of trends in cancer survival 2000-14 (CONCORD-3): analysis of individual records for 37 513025 patients diagnosed with one of 18 cancers from 322 population-based registries in 71 countries. LANCET 391:1023-1075. 10.1016/S0140-6736(17)33326-3

[2]. Bray, F., Ferlay, J., Soerjomataram, I., Siegel, R.L., Torre, L.A., and Jemal, A. 2018. Global cancer statistics 2018: GLOBOCAN estimates of incidence and mortality worldwide for 36 cancers in 185 countries. CA Cancer J Clin 68:394-424. 10.3322/caac.21492

[3]. Brambilla, E., Travis, W.D., Colby, T.V., Corrin, B., and Shimosato, Y. 2001. The new World Health Organization classification of lung tumours. EUROPEAN RESPIRATORY JOURNAL 18:1059-1068.

[4]. Wang, L.C., Wang, L., Kwauk, S., Woo, J.A., Wu, L.Q., Zhu, H., Zhan, L.Z., Sun, N.L., and Zhang, L. 2011. Analysis on the clinical features of 22 basaloid squamous cell carcinoma of the lung. Journal of Cardiothoracic Surgery 6:10. 10.1186/1749-8090-6-10

[5]. Crapanzano, J.P., Loukeris, K., Borczuk, A.C., and Saqi, A. 2011. Cytological, histological, and immunohistochemical findings of pulmonary carcinomas with basaloid features. DIAGNOSTIC CYTOPATHOLOGY 39:92-100. 10.1002/dc.21335

[6]. Travis, W.D., Brambilla, E., Nicholson, A.G., Yatabe, Y., Austin, J., Beasley, M.B., Chirieac, L.R., Dacic, S., Duhig, E., Flieder, D.B., Geisinger, K., Hirsch, F.R., Ishikawa, Y., Kerr, K.M., Noguchi, M., Pelosi, G., Powell, C.A., Tsao, M.S., and Wistuba, I. 2015. The 2015 World Health Organization Classification of Lung Tumors: Impact of Genetic, Clinical and Radiologic Advances Since the 2004 Classification. Journal of Thoracic Oncology 10:1243-1260. 10.1097/JTO.0000000000000630

[7].Vignaud, J.M. 2016. [Squamous cell carcinoma, basaloid squamous cell carcinoma and adenosquamous carcinoma in the lung]. ANNALES DE PATHOLOGIE 36:15-23. 10.1016/j.annpat.2015.11.011 
221 [8]. Sun, F., Ma, K., Yang, X., Li, M., Shi, Y., Zhan, C., Jiang, W., and Wang, Q. 2017. A nomogram to

222

223

224

225

226

227

228

229

230

231

232

233

234

235

236

237

238

239

240

241

242

243

244

245

246

247

248

249

250

251

252

253

254

255

256

257

258

259

260

261

predict prognosis after surgery in early stage non-small cell lung cancer in elderly patients. International Journal of Surgery 42:11-16. 10.1016/j.ijsu.2017.04.024

[9]. Wang, S., Ma, K., Chen, Z., Yang, X., Sun, F., Jin, Y., Shi, Y., Jiang, W., Wang, Q., and Zhan, C. 2018. A Nomogram to Predict Prognosis in Malignant Pleural Mesothelioma. WORLD JOURNAL OF SURGERY 42:2134-2142. 10.1007/s00268-017-4424-6

[10]. Kim, D.J., Kim, K.D., Shin, D.H., Ro, J.Y., and Chung, K.Y. 2003. Basaloid carcinoma of the lung: a really dismal histologic variant? ANNALS OF THORACIC SURGERY 76:1833-1837.

[11]. Brambilla, E., Moro, D., Veale, D., Brichon, P.Y., Stoebner, P., Paramelle, B., and Brambilla, C. 1992. Basal cell (basaloid) carcinoma of the lung: a new morphologic and phenotypic entity with separate prognostic significance. HUMAN PATHOLOGY 23:993-1003.

[12]. Brambilla, C., Laffaire, J., Lantuejoul, S., Moro-Sibilot, D., Mignotte, H., Arbib, F., Toffart, A.C., Petel, F., Hainaut, P., Rousseaux, S., Khochbin, S., de Reynies, A., and Brambilla, E. 2014. Lung squamous cell carcinomas with basaloid histology represent a specific molecular entity. CLINICAL CANCER RESEARCH 20:5777-5786. 10.1158/1078-0432.CCR-14-0459

[13]. Moro-Sibilot, D., Lantuejoul, S., Diab, S., Moulai, N., Aubert, A., Timsit, J.F., Brambilla, C., Brichon, P.Y., and Brambilla, E. 2008. Lung carcinomas with a basaloid pattern: a study of 90 cases focusing on their poor prognosis. EUROPEAN RESPIRATORY JOURNAL 31:854-859. 10.1183/09031936.00058507

[14]. Moro, D., Brichon, P.Y., Brambilla, E., Veale, D., Labat, F., and Brambilla, C. 1994. Basaloid bronchial carcinoma. A histologic group with a poor prognosis. CANCER 73:2734-2739.

[15]. Lang-Lazdunski, L. 2013. Surgery for nonsmall cell lung cancer. Eur Respir Rev 22:382-404. $10.1183 / 09059180.00003913$

[16]. Zhang, H., and Shen-Tu, Y. 2015. [Clinical Advance of Sublobectomy for Early Stage Non-small Cell Lung Cancer]. Zhongguo Fei Ai Za Zhi 18:565-570. 10.3779/j.issn.1009-3419.2015.09.07

[17]. Wang, J., and Zhao, H. 2016. [Issues Need to be Considered in Sublobectomy for Early Stage Lung Cancer]. Zhongguo Fei Ai Za Zhi 19:351-354. 10.3779/j.issn.1009-3419.2016.06.10

[18]. Zhang, Y., Sun, Y., Wang, R., Ye, T., Zhang, Y., and Chen, H. 2015. Meta-analysis of lobectomy, segmentectomy, and wedge resection for stage I non-small cell lung cancer. JOURNAL OF SURGICAL ONCOLOGY 111:334-340. 10.1002/jso.23800

[19]. Kent, M.S., Mandrekar, S.J., Landreneau, R., Nichols, F., Foster, N.R., DiPetrillo, T.A., Meyers, B., Heron, D.E., Jones, D.R., Tan, A.D., Starnes, S., Putnam, J.J., and Fernando, H.C. 2016. A Nomogram to Predict Recurrence and Survival of High-Risk Patients Undergoing Sublobar Resection for Lung Cancer: An Analysis of a Multicenter Prospective Study (ACOSOG Z4032). ANNALS OF THORACIC SURGERY 102:239-246. 10.1016/j.athoracsur.2016.01.063

[20]. Zhang, J., Gold, K.A., Lin, H.Y., Swisher, S.G., Xing, Y., Lee, J.J., Kim, E.S., and William, W.J. 2015. Relationship between tumor size and survival in non-small-cell lung cancer (NSCLC): an analysis of the surveillance, epidemiology, and end results (SEER) registry. Journal of Thoracic Oncology 10:682-690. 10.1097/JTO.0000000000000456

[21]. Zhang, B., Yuan, Z., Zhao, L., Pang, Q., and Wang, P. 2017. Nomograms for predicting progression and efficacy of post-operation radiotherapy in IIIA-pN2 non-small cell lung cancer patients. Oncotarget 8:3720837216. 10.18632/oncotarget. 16564 
262 [22]. Young, K.A., Efiong, E., Dove, J.T., Blansfield, J.A., Hunsinger, M.A., Wild, J.L., Shabahang, M.M., 263 and Facktor, M.A. 2017. External Validation of a Survival Nomogram for Non-Small Cell Lung Cancer Using 264 the National Cancer Database. ANNALS OF SURGICAL ONCOLOGY 24:1459-1464. 10.1245/s10434-017$265 \quad 5795-5$

266 [23]. Ye, L., Shi, S., Zeng, Z., Huang, Y., Hu, Y., and He, J. 2018. Nomograms for predicting disease 267 progression in patients of Stage I non-small cell lung cancer treated with stereotactic body radiotherapy. 268 JAPANESE JOURNAL OF CLINICAL ONCOLOGY 48:160-166. 10.1093/jjco/hyx179

269 [24]. Liang, W., Zhang, L., Jiang, G., Wang, Q., Liu, L., Liu, D., Wang, Z., Zhu, Z., Deng, Q., Xiong, X., Shao, 270 W., Shi, X., and He, J. 2015. Development and validation of a nomogram for predicting survival in patients 271 with resected non-small-cell lung cancer. JOURNAL OF CLINICAL ONCOLOGY 33:861-869. $27210.1200 / \mathrm{JCO} .2014 .56 .6661$

273 [25]. Xie, D., Marks, R., Zhang, M., Jiang, G., Jatoi, A., Garces, Y.I., Mansfield, A., Molina, J., and Yang, P. 274 2015. Nomograms Predict Overall Survival for Patients with Small-Cell Lung Cancer Incorporating 275 Pretreatment Peripheral Blood Markers. Journal of Thoracic Oncology 10:1213-1220. $27610.1097 / J T O .0000000000000585$

277 [26]. Keedy, V.L., Temin, S., Somerfield, M.R., Beasley, M.B., Johnson, D.H., McShane, L.M., Milton, D.T., 278 Strawn, J.R., Wakelee, H.A., and Giaccone, G. 2011. American Society of Clinical Oncology provisional 279 clinical opinion: epidermal growth factor receptor (EGFR) Mutation testing for patients with advanced non280 small-cell lung cancer considering first-line EGFR tyrosine kinase inhibitor therapy. JOURNAL OF 281 CLINICAL ONCOLOGY 29:2121-2127. 10.1200/JCO.2010.31.8923

282 [27]. Felip, E., Gridelli, C., Baas, P., Rosell, R., and Stahel, R. 2011. Metastatic non-small-cell lung cancer: 283 consensus on pathology and molecular tests, first-line, second-line, and third-line therapy: 1st ESMO 284 Consensus Conference in Lung Cancer; Lugano 2010. ANNALS OF ONCOLOGY 22:1507-1519. 285 10.1093/annonc/mdr150

286 [28]. Yang, X., Sun, F., Chen, L., Shi, M., Shi, Y., Lin, Z., Feng, M., Zhan, C., Jiang, W., and Wang, Q. 2017. 287 Prognostic value of visceral pleural invasion in non-small cell lung cancer: A propensity score matching study 288 based on the SEER registry. JOURNAL OF SURGICAL ONCOLOGY 116:398-406. 10.1002/jso.24677

289 [29]. Yang, X., Zhan, C., Li, M., Huang, Y., Zhao, M., Yang, X., Lin, Z., Shi, Y., Jiang, W., and Wang, Q. 290 2018. Lobectomy Versus Sublobectomy in Metachronous Second Primary Lung Cancer: A Propensity Score 291 Study. ANNALS OF THORACIC SURGERY 106:880-887. 10.1016/j.athoracsur.2018.04.071 


\section{Figure legends}

307

308

309

Figure 1 The flow diagram of the selection process for the study cohort.

Figure 2 The survival curve of basaloid squamous cell carcinoma of the lung (BSC), lung squamous cell carcinoma (SCC), large cell carcinoma (LCC) and lung adenocarcinoma (LAC).

Figure 3 (A) Survival analyses for patients with different surgeries and without surgery. (B) Survival analyses for patients with lobectomy and sublobar resection classified by tumor histology and tumor stage. (C) Stage I (1-, 3-, 5-year survival rate of lobectomy vs. sublobar section: $82.0 \%, 53.2 \%, 31.5 \%$ vs. $70.7 \%, 46.3 \%, 17.1 \%$ ). (D) Stage II (1-, 3-, 5-year survival rate of lobectomy vs. sublobar section : 71.1\%, 40.0\%, 31.1\% vs. 75.0\%, $12.5 \%, 12.5 \%)$. (E) Stage III and IV (1-, 3-, 5-year survival rate of lobectomy vs. sublobar section: 67.4\%, $32.6 \%, 18.6 \%$ vs. $46.2 \%, 7.7 \%, 0.0 \%)$.

Figure 4 (A) Nomogram for prediction of 1-, 3- and 5- year overall survival of patients with basaloid squamous cell carcinoma of the lung (BSC). Calibration plots of the nomogram prediction of (B) 1-year, (C) 3year and (D) 5-year overall survival nomogram calibration curves. The red line represents equality of the observed and predicted probability.

\section{Supplementary material}

Figure 5 (A) The survival curve of basaloid squamous cell carcinoma of the lung (BSC) and lung squamous 
322 cell carcinoma (SCC). (B) The survival curve of basaloid squamous cell carcinoma of the lung (BSC) and

323 large cell carcinoma (LCC). (C) The survival curve of basaloid squamous cell carcinoma of the lung (BSC)

324 and lung adenocarcinoma (LAC).

325 Figure 6 The survival curve of poor differentiated basaloid squamous cell carcinoma of the lung (PBSC) and 326 poor differentiated lung squamous cell carcinoma (PSCC).

327

328 


\section{Table $\mathbf{1}$ (on next page)}

Comparison of the clinipathological characteristics of basaloid squamous cell carcinoma (BSC), squamous cell carcinoma (SCC), large cell carcinoma (LCC) and lung adenocarcinoma (LAC).

${ }^{*} \mathrm{P}$ value for chi-square test. BSC, Basaloid squamous cell carcinoma; SCC, squamous cell carcinoma; LCC $\square$ large cell carcinoma $\square$ LAC $\square$ lung adenocarcinoma $\square$ IQR, interquartile range. 
Table 1. Comparison of the clinipathological characteristics of basaloid squamous cell carcinoma (BSC), squamous cell carcinoma of the lung (SCC), large cell carcinoma of the lung (LCC) and lung adenocarcinoma (LAC).

\begin{tabular}{|c|c|c|c|c|c|c|c|}
\hline Characteristics & $\mathrm{BSC}$ & $\mathrm{SCC}$ & $\mathrm{p}$ value & $\mathrm{LCC}$ & $\mathrm{p}$ value & LAC & $\mathrm{p}$ value \\
\hline Race, $n(\%)$ & & & 0.351 & & 0.039 & & 0.049 \\
\hline White & $358(84.2)$ & $75272(83.6)$ & & $5682(81.2)$ & & $128685(80.1)$ & \\
\hline Black & $43(10.1)$ & $10617(11.8)$ & & $999(14.3)$ & & 18644(11.6) & \\
\hline Other & $24(5.7)$ & $4117(4.6)$ & & $316(4.5)$ & & $13309(8.3)$ & \\
\hline Age, median [IQR] & $\begin{array}{l}70.15 \\
(59.87-80.43)\end{array}$ & $\begin{array}{l}70.41 \\
(60.66-80.16)\end{array}$ & 0.139 & $\begin{array}{l}67.92 \\
(56.87-78.97)\end{array}$ & 0.303 & $\begin{array}{l}68.37 \\
(57.27-79.47)\end{array}$ & 0.376 \\
\hline Sex, n (\%) & & & 0.503 & & 0.443 & & $<0.001$ \\
\hline Male & $257(60.5)$ & $55849(62.1)$ & & $4099(58.6)$ & & $78527(48.9)$ & \\
\hline Female & $168(39.5)$ & $34157(37.9)$ & & $2898(41.4)$ & & $82111(51.1)$ & \\
\hline Grade, n (\%) & & & $<0.001$ & & $<0.001$ & & $<0.001$ \\
\hline Well differetiated & $2(0.5)$ & $1974(2.2)$ & & $15(0.2)$ & & $11955(7.4)$ & \\
\hline Moderately differetiated & $46(10.8)$ & $26606(29.6)$ & & $80(1.1)$ & & $36005(22.4)$ & \\
\hline Poorly differetiated & $274(64.5)$ & $32307(35.9)$ & & $2115(30.2)$ & & $43291(27.0)$ & \\
\hline Undifferetiated & $13(3.0)$ & $648(0.7)$ & & $2299(32.9)$ & & $968(0.6)$ & \\
\hline Unknown & $90(21.2)$ & 28471(31.6) & & $2488(35.6)$ & & $68419(42.6)$ & \\
\hline Total stage,n (\%) & & & $<0.001$ & & $<0.001$ & & $<0.001$ \\
\hline $\mathrm{I}$ & $179(42.1)$ & $27683(30.8)$ & & $1460(20.9)$ & & $41408(25.8)$ & \\
\hline II & $63(14.8)$ & $10946(12.2)$ & & $534(7.6)$ & & $10560(6.6)$ & \\
\hline III & $101(23.8)$ & $26297(29.2)$ & & $1858(26.6)$ & & $32528(20.2)$ & \\
\hline IV & $82(19.3)$ & $25080(27.8)$ & & $3145(44.9)$ & & 76142(47.4) & \\
\hline T stage, n (\%) & & & $<0.001$ & & $<0.001$ & & 0.001 \\
\hline $\mathrm{T} 1$ & $133(31.3)$ & $9151(10.2)$ & & $1292(10.2)$ & & $43558(27.1)$ & \\
\hline $\mathrm{T} 2$ & $139(32.7)$ & $66275(73.6)$ & & $2346(73.6)$ & & $47776(29.8)$ & \\
\hline $\mathrm{T} 3$ & $68(16.0)$ & $3596(4.0)$ & & $880(4.0)$ & & $22072(13.7)$ & \\
\hline $\mathrm{T} 4$ & $85(20.0)$ & $10984(12.2)$ & & $2479(12.2)$ & & $47232(29.4)$ & \\
\hline
\end{tabular}




\begin{tabular}{|c|c|c|c|c|c|c|c|}
\hline N stage, $n(\%)$ & & & $<0.001$ & & $<0.001$ & & $<0.001$ \\
\hline No & $269(63.3)$ & $9151(10.2)$ & & $2847(40.7)$ & & $72104(44.9)$ & \\
\hline N1 & $50(11.8)$ & $66275(73.6)$ & & $663(9.5)$ & & $14110(8.8)$ & \\
\hline N2 & $84(19.8)$ & $3596(4.0)$ & & $2628(37.5)$ & & $54487(33.9)$ & \\
\hline N3 & $22(5.1)$ & $10984(12.2)$ & & $859(12.3)$ & & $19937(12.4)$ & \\
\hline M stage,n (\%) & & & $<0.001$ & & $<0.001$ & & $<0.001$ \\
\hline M0 & $343(80.7)$ & $58972(65.5)$ & & $3854(55.1)$ & & $84496(52.6)$ & \\
\hline M1 & $82(19.3)$ & $31034(34.5)$ & & $3143(44.9)$ & & $76142(47.4)$ & \\
\hline Surgery, n (\%) & & & $<0.001$ & & $<0.001$ & & $<0.001$ \\
\hline Not performed & $144(33.9)$ & $60336(67.0)$ & & $5041(72.0)$ & & $112857(70.3)$ & \\
\hline Lobectomy & $199(46.8)$ & $20978(23.3)$ & & $1377(19.7)$ & & $35329(22.0)$ & \\
\hline Sublobar resection & $62(14.6)$ & $6153(6.8)$ & & $438(6.3)$ & & $10982(6.8)$ & \\
\hline Pneumonectomy & $20(4.7)$ & $2539(2.8)$ & & $141(2.0)$ & & $1470(0.9)$ & \\
\hline Radiotherapy, n (\%) & & & $<0.001$ & & $<0.001$ & & $<0.001$ \\
\hline No & $104(24.5)$ & $4(0.0)$ & & $3980(56.9)$ & & $98823(61.5)$ & \\
\hline Yes & $321(75.5)$ & 90002 & & $3017(43.1)$ & & $61815(38.5)$ & \\
\hline & & $(100.0)$ & & & & & \\
\hline Chemotherapy, n (\%) & & & $<0.001$ & & $<0.001$ & & $<0.001$ \\
\hline No & $134(31.5)$ & $53403(59.3)$ & & $3944(56.4)$ & & $88220(54.9)$ & \\
\hline Yes & $291(68.5)$ & $36603(40.7)$ & & $3053(43.6)$ & & $72418(45.1)$ & \\
\hline
\end{tabular}

BSC, Basaloid squamous cell carcinoma; SCC, squamous cell carcinoma; LCC, large cell carcinoma of the lung; LAC, lung adenocarcinoma; IQR, interquartile range. 
Table 2 (on next page)

Univariate and multivariate Cox proportional hazards analysis of BSC.

${ }^{*} \mathrm{P}$ value for chi-square test. C-index $=0.750$. 
1 Table 2. Univariate and multivariate Cox proportional hazards analysis

\begin{tabular}{|c|c|c|c|c|}
\hline \multirow[t]{2}{*}{ Patient characteristics } & \multicolumn{2}{|c|}{ Univariate analysis } & \multicolumn{2}{|c|}{ Multivariate analysis } \\
\hline & $\operatorname{HR}(95 \% \mathrm{CI})$ & $p$ value & $\operatorname{HR}(95 \% \mathrm{CI})$ & $\mathrm{p}$ value \\
\hline Race & & 0.077 & & - \\
\hline White & Reference & & - & - \\
\hline Black & $1.251(0.811-1.931)$ & 0.311 & - & - \\
\hline Other & $0.502(0.248-1.019)$ & 0.056 & - & - \\
\hline Age & & $<0.001$ & $1.032(1.017-1.048)$ & $<0.001$ \\
\hline Sex & & 0.257 & & - \\
\hline Male & Reference & & - & - \\
\hline Female & $0.855(0.652-1.121)$ & & - & - \\
\hline Grade & & $<0.001$ & & 0.248 \\
\hline Well differetiated & Reference & & Reference & \\
\hline Moderately differetiated & $0.776(0.181-3.322)$ & 0.733 & $2.179(0.404-11.756)$ & 0.365 \\
\hline Poorly differetiated & $0.994(0.245-4.033)$ & 0.993 & $1.894(0.369-9.712)$ & 0.444 \\
\hline Undifferetiated & $1.748(0.375-8.139)$ & 0.477 & $4.198(0.689-25.591)$ & 0.120 \\
\hline Unknown & $2.291(0.537-9.421)$ & 0.251 & $2.101(0.401-10.988)$ & 0.379 \\
\hline AJCC 8th T stage & & $<0.001$ & & $<0.001$ \\
\hline $\mathrm{T} 1$ & Reference & & Reference & \\
\hline $\mathrm{T} 2$ & $1.052(0.735-1.507)$ & 0.780 & $1.240(0.856-1.797)$ & 0.256 \\
\hline $\mathrm{T} 3$ & $1.909(1.274-2.862)$ & 0.002 & $1.935(1.257-2.980)$ & 0.003 \\
\hline $\mathrm{T} 4$ & $2.689(1.879-3.849)$ & $<0.001$ & $2.364(1.559-3.585)$ & $<0.001$ \\
\hline AJCC 8th N stage & & $<0.001$ & & 0.009 \\
\hline No & Reference & & Reference & \\
\hline N1 & $1.678(1.088-2.587)$ & 0.019 & $1.623(1.018-2.586)$ & 0.042 \\
\hline $\mathrm{N} 2$ & $3.218(2.360-4.390)$ & $<0.001$ & $1.905(1.300-2.792)$ & 0.001 \\
\hline N3 & $5.013(2.855-8.804)$ & $<0.001$ & $1.826(0.951-3.505)$ & 0.071 \\
\hline AJCC 8th M stage & & $<0.001$ & & $<0.001$ \\
\hline
\end{tabular}




\begin{tabular}{|c|c|c|c|c|}
\hline M0 & Reference & & Reference & \\
\hline M1 & $4.253(3.170-5.704)$ & $<0.001$ & $2.399(1.695-3.393)$ & $<0.001$ \\
\hline Surgery & & $<0.001$ & & $<0.001$ \\
\hline Not performed & Reference & & Reference & \\
\hline Lobectomy & $0.187(0.136-0.258)$ & $<0.001$ & $0.389(0.263-0.578)$ & $<0.001$ \\
\hline Sublobar resection & $0.442(0.304-0.643)$ & $<0.001$ & $0.889(0.567-1.394)$ & 0.608 \\
\hline Pneumonectomy & $0.384(0.209-0.706)$ & $<0.001$ & $0.614(0.317-1.189)$ & 0.148 \\
\hline Radiotherapy & & $<0.001$ & & \\
\hline No & Reference & & Reference & \\
\hline Yes & $2.173(1.631-2.895)$ & $<0.001$ & $1.076(0.768-1.508)$ & 0.669 \\
\hline \multicolumn{5}{|l|}{ Chemotherapy } \\
\hline No & Reference & & Reference & \\
\hline Yes & $1.423(1.076-1.882)$ & 0.013 & $0.725(0.506-1.039)$ & 0.080 \\
\hline
\end{tabular}

3 The total stage was not an independent variable related to T, N, and M stage, therefore it was excluded in the multivariate analysis. 
Figure 1

The flow diagram of the selection process for the study cohort.

The flow diagram of the selection process for the study cohort.

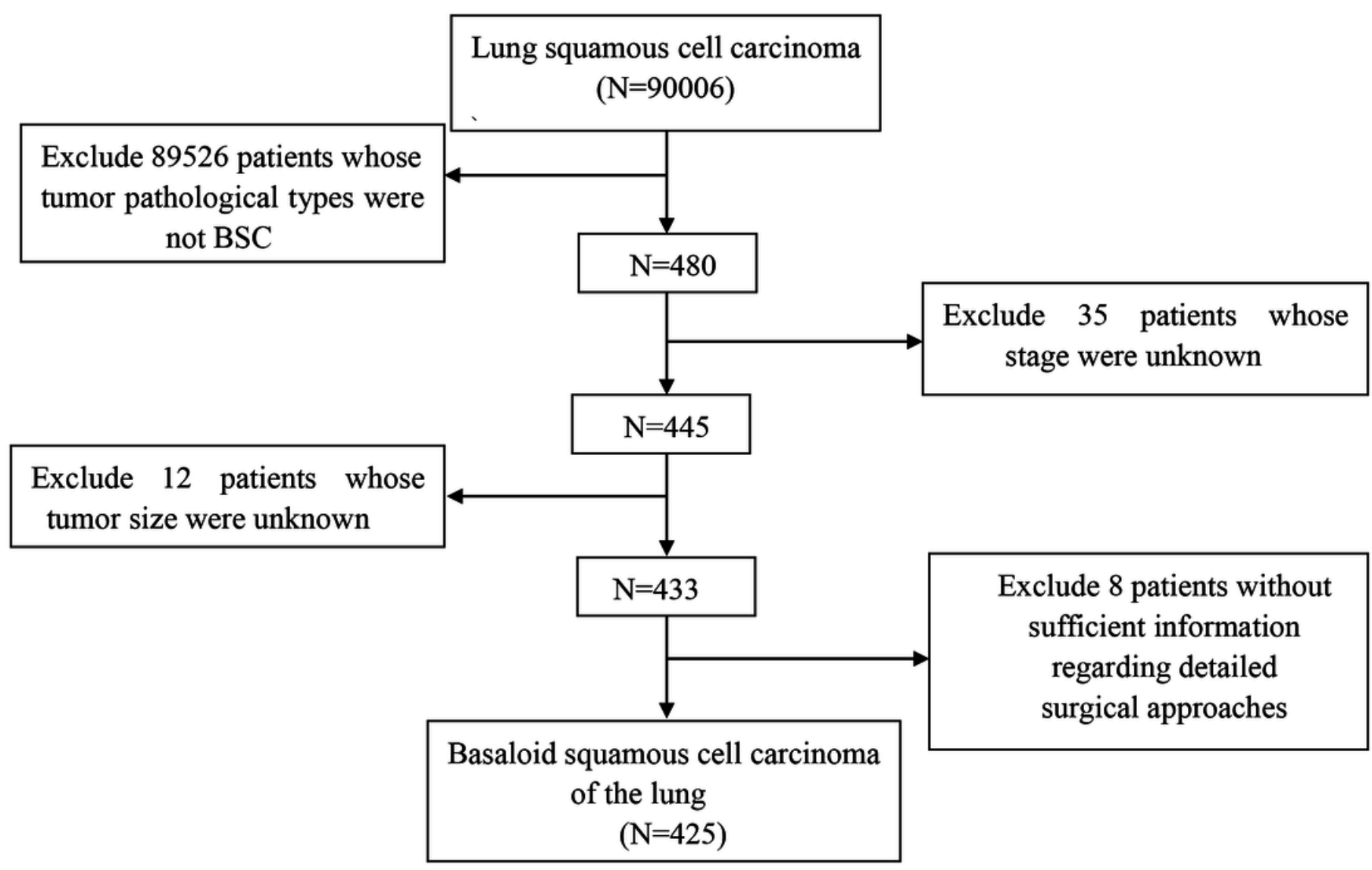


Figure 2

Survival for BSC $\square \mathrm{SCC} \square \mathrm{LCC}$ and LAC

The survival curves of basaloid squamous cell carcinoma of the lung (BSC) $\square$ squamous cell carcinoma (SCC) $]$ large cell carcinoma (LCC) and lung adenocarcinoma (LAC) . 


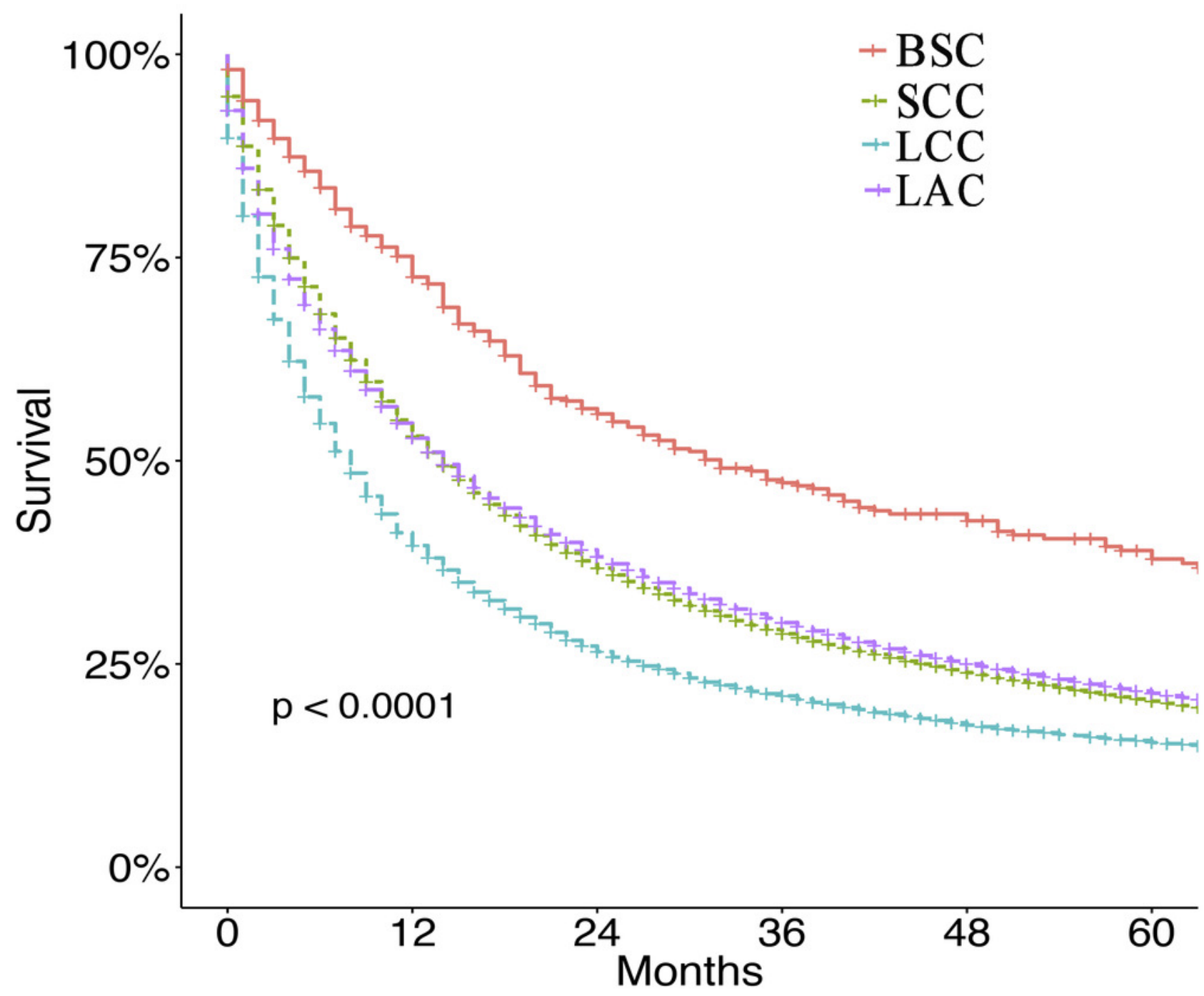

Patients at risk

\begin{tabular}{|c|c|c|c|c|c|c|}
\hline BSC & 425 & 266 & 175 & 130 & 104 & 75 \\
\hline $\mathrm{SCC}$ & 90006 & 44210 & 26900 & 18399 & 13298 & 9658 \\
\hline $\mathrm{LCC}$ & 6997 & 2796 & 1789 & 1351 & 1071 & 885 \\
\hline $\mathrm{LAC}$ & 60482 & 77177 & 48437 & 32991 & 23365 & 16747 \\
\hline
\end{tabular}




\section{Figure 3}

survival for surgical modalities

(A) Survival analyses for patients with different surgeries and without surgery. (B) Survival analyses for patients with lobectomy and sublobar resection classified by tumor histology and tumor stage. (C) Stage I (1-, 3-, 5-year survival rate of lobectomy vs. sublobar section: $82.0 \%, 53.2 \%, 31.5 \%$ vs. $70.7 \%, 46.3 \%, 17.1 \%)$. (D) Stage II (1-, 3-, 5-year survival rate of lobectomy vs. sublobar section : $71.1 \%, 40.0 \%, 31.1 \%$ vs. $75.0 \%, 12.5 \%, 12.5 \%)$. (E) Stage III and IV (1-, 3-, 5-year survival rate of lobectomy vs. sublobar section: $67.4 \%, 32.6 \%, 18.6 \%$ vs. $46.2 \%, 7.7 \%, 0.0 \%)$.
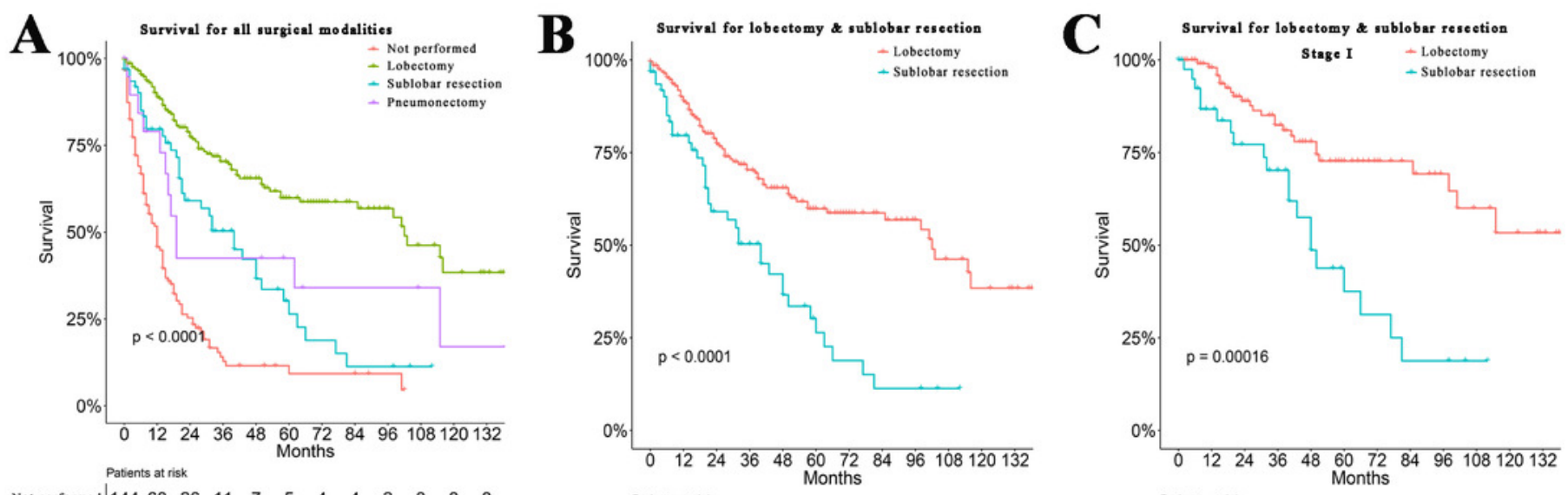

ot performed $144 \begin{array}{llllllllllll}60 & 26 & 11 & 7 & 5 & 4 & 4 & 2 & 0 & 0 & 0\end{array}$

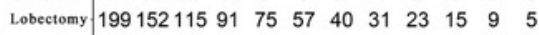
Sublobar resection. $\begin{array}{llllllllllll}62 & 41 & 27 & 21 & 15 & 8 & 5 & 3 & 3 & 1 & 0 & 0\end{array}$

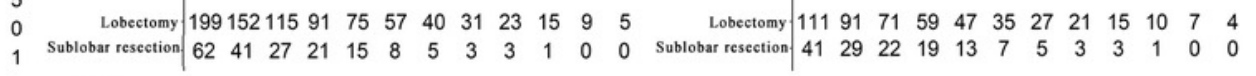
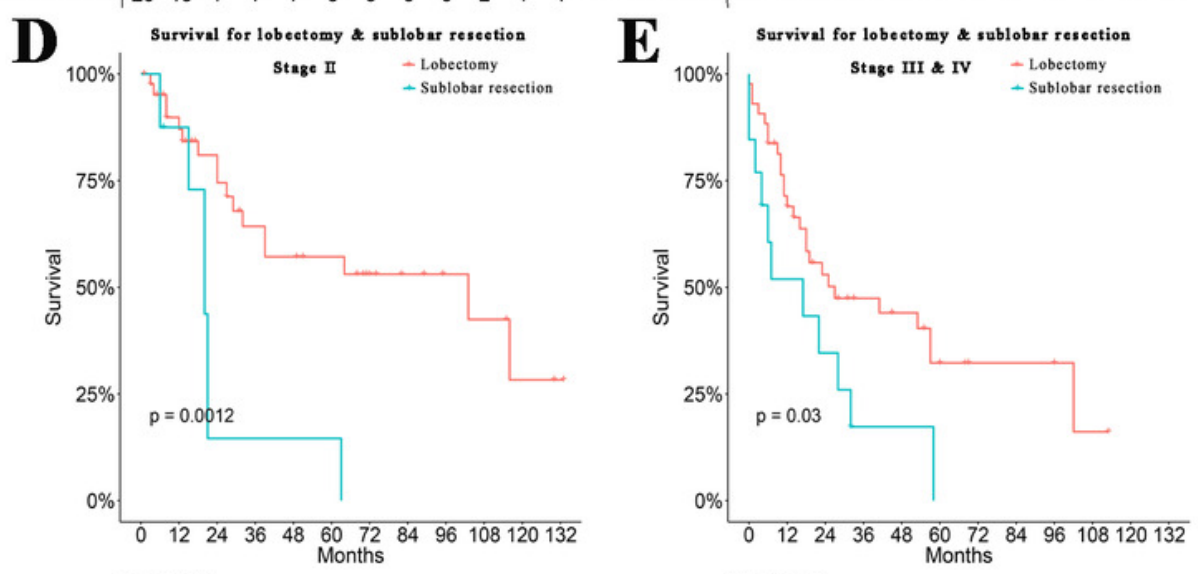

\begin{tabular}{l|lllllllllllll} 
Lobectomy & 45 & 32 & 25 & 18 & 16 & 14 & 10 & 7 & 5 & 4 & 2 & 1
\end{tabular}

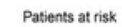

Lobectomy $\mid \begin{array}{lllllllllllll}43 & 29 & 19 & 14 & 12 & 8 & 3 & 3 & 3 & 1 & 0 & 0\end{array}$

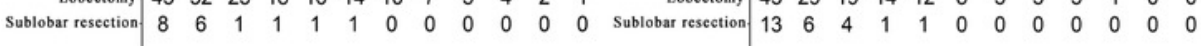




\section{Figure 4}

Nomogram and overall survival nomogram calibration curves.

(A) Nomogram for prediction of 1-, 3- and 5-year overall survival of patients with basaloid squamous cell carcinoma of the lung (BSC). Calibration plots of the nomogram prediction of (B) 1-year,(C) 3-year and (D) 5-year overall survival nomogram calibration curves. The red line represents equality of the observed and predicted probability.

$\mathbf{A}$

Points

$10 \quad 20$

30

40

60

70

80

90

100

Age

Tstage

Nstage

Mstage

Surgery

Total Points

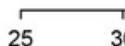

$\begin{array}{lllll}30 & 35 & 40 & 45 & 50\end{array}$ T4

$\begin{array}{llll}\mathrm{T} 1 & \mathrm{~T} 3 & \mathrm{~N} 1 & \\ & & \mathrm{~N} 3\end{array}$

1-year survival

3-year survival

5-year survival

\begin{tabular}{|c|c|c|c|c|c|c|c|c|c|}
\hline 0.9 & & 0.8 & 0.7 & 0.6 & 0.5 & 0.4 & 0.3 & 0.2 & $0.1 \quad 0.05$ \\
\hline & 0.8 & 0.7 & 0.6 & 0.5 & 0.4 & 0.3 & 0.2 & 0.1 & 0.05 \\
\hline
\end{tabular}
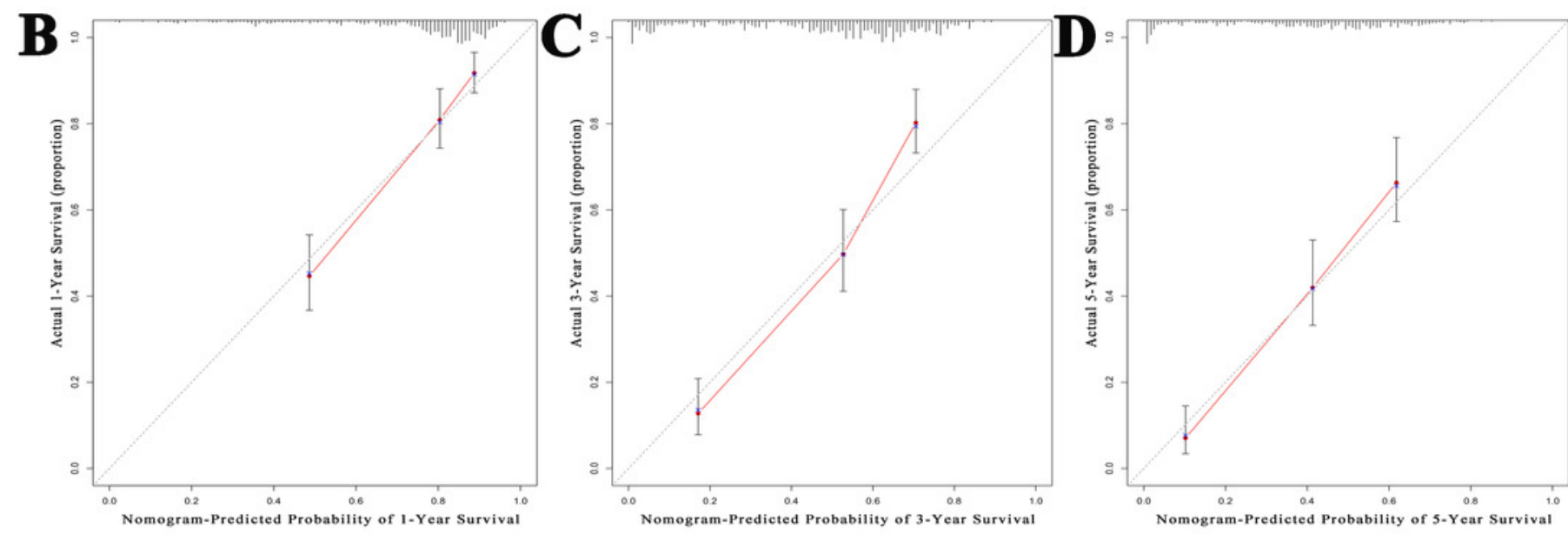